\title{
Quels taux de détection des adénomes est synonyme de coloscopie de qualité et efficacement préventive ?
}

\author{
What Adenoma Detection Rate is Synonymous with Well-Performed \\ and Effectively Preventive Colonoscopy?
}

\author{
D. Heresbach \\ (C) Springer-Verlag France 2014
}

Le taux de détection des adénomes (TDA) est un critère de qualité reconnu, puisque c'est le but principal de la coloscopie. Il avait été montré que le taux de cancer colorectal (CCR) d'intervalle survenu six mois après une coloscopie était significativement plus élevé en cas de TDA inférieur à $20 \%$ comparé à celui supérieur à $20 \%$ : cette étude n'avait pas montré quel était le taux requis de TDA (au-delà de $20 \%$ ) pour diminuer le taux de CCR d'intervalle. Différentes hypothèses étaient émises, mais toujours dans des sous-populations particulières, notamment après recherche de saignement occulte dans les selles, filtre qui artificiellement augmentait le TDA, quelle que soit la qualité de la coloscopie.

L'étude de Corley et al. [1] relate, à partir des résultats d'un programme assurantiel de dépistage et prévention par coloscopie totale, le TDA et le taux des CCR d'intervalle. L'analyse est basée sur 314872 coloscopies de dépistage réalisées par 136 gastroentérologues. Pendant un suivi médian de 35 mois, 712 CCR d'intervalle sont survenus au moins six mois après la coloscopie index. L'analyse de ces CCR d'intervalle a été réalisée en fonction du TDA divisé en cinq classes ; 7-19\%, 19-24\%, 24-28\%, 28-33\% et $33-52 \%$, soit en cinq quintiles. Le taux de CCR d'intervalle, de CCR avancé ou de décès par CCR était corrélé au TDA. Ce résultat n'est pas nouveau, mais cette large étude précise, lorsque le TDA est supérieur à $20 \%$, à quel niveau il doit se situer pour diminuer significativement le taux de CCR d'intervalle.
Un TDA moyen de $31 \%$ avec un intervalle de confiance (IC) de 28,4 à 33,5\% est associé à un taux de CCR d'intervalle, CCR avancé ou CCR mortel significativement diminué respectivement de $30 \%$ (IC 95\%: [9-36]), de $42 \%$ (IC $95 \%$ : [29-67]) et de $49 \%$ (IC $95 \%$ : [19-67]) par rapport à un TDA inférieur à $20 \%$.

Au total, un TDA de $20 \%$ est bien, mais un taux de $28 \%$ c'est la signature d'une coloscopie non seulement de qualité mais efficace. Ce message est essentiel, et pour obtenir le «permis de coloscopie préventive », nous devrions individuellement prouver préalablement l'obtention de ce seuil. Pourquoi ne pas l'inclure dans un pacte de responsabilité ?

Conflit d'intérêt : Le Pr Denis Heresbach a réalisé des missions ponctuelles d'expert auprès des laboratoires Norgine, Aptalis en 2010 et de la société MedPass, Norgine et Aptalis en 2011. Il a participé à un symposium organisé par MaunaKea Technology en 2010, Ella SA en 2011 et au Club francophone d'échoendoscopie (CFE) de 2012 avec le soutien de Wilson-Cook France.

\section{Référence}

1. Corley DA, Jensen CD, Marks AR, Zhao WK, Lee JK, Doubeni CA, et al. Adenoma detection rate and risk of colorectal cancer and death. New Engl J Med 2014; 370:1298-306.

\footnotetext{
D. Heresbach $(\square)$

Unité d'endoscopie et de consultation digestive, centre hospitalier de Redon, 8, rue Étienne-Gascon, F-35603 Redon, France

e-mail : Denis.heresebach@ch-redon.fr
} 